\title{
Efficacy and safety of a new low-volume PEG with citrate and simethicone bowel preparation for colonoscopy (Clensia): a multicenter randomized observer-blind clinical trial vs. a low-volume PEG with ascorbic acid (PEG-ASC)
}

\section{다(1)우우}

\section{Authors}

Patrizia Kump ${ }^{1}$, Cesare Hassan², Cristiano Spada ${ }^{3,4}$, Eva Brownstone ${ }^{5}$, Christian Datz ${ }^{6}$, Michael Haefner ${ }^{7}$, Friedrich Renner $^{8}$, Rainer Schoefl ${ }^{9}$, Florian Schreiber ${ }^{1}$

Institutions

1 Department of Internal Medicine, Division of Gastroenterology and Hepatology, Medical University Graz, Graz, Austria

2 Gastroenterology Department, Nuovo Regina Margherita Hospital, Rome, Italy

3 Digestive Endoscopy Unit, Catholic University, Rome, Italy

4 Digestive Endoscopy Unit, Fondazione Poliambulanza, Brescia, Italy

5 Internal Medicine IV, KH Rudolfstiftung, Vienna, Austria

6 Department of Internal Medicine, Oberndorf Hospital, Teaching Hospital of the Paracelsus Medical University, Salzburg, Austria

7 Department of Gastroenterology and Hepatology, Medical University of Vienna, Vienna, Austria

8 Interne Abteilung, Krankenhaus der Barmherzigen Schwestern Ried, Ried, Austria

9 Internal Medicine IV, KH der Elisabethinen, Linz, Austria.

submitted 20.11.2017

accepted after revision 5.3.2018

Bibliography

DOI https://doi.org/10.1055/a-0624-2266 |

Endoscopy International Open 2018; 06: E907-E913

(c) Georg Thieme Verlag KG Stuttgart · New York ISSN 2364-3722

Corresponding author

Cristiano Spada, MD, PhD, Digestive Endoscopy Unit, Fondazione Poliambulanza, Via Bissolati 57, 25124 Brescia (Italia)

Fax: +390630157220

cristiano.spada@poliambulanza.it

\section{ABSTRACT}

Background and study aims Quality of inspection during colonoscopy is strictly related to the level of cleansing. High-volume (PEG-based) solutions are highly effective and safe, but their high volume affects tolerability and compliance. The aim of this study was to compare a new low-volume PEG with citrate and simethicone solution (PMF 104,Clensia) with a low-volume PEG with ascorbic acid solution (PEG-ASC; Moviprep).

Patients and methods This was a multicenter, randomized, observer-blind, parallel-group, phase 3 clinical trial, where patients were randomized between PMF 104 and PEG-ASC. In both groups, patients were instructed to take a full-dose regimen the evening before if colonoscopy was scheduled before $11 \mathrm{am}$ to $12 \mathrm{pm}$, or to take a split regimen if colonoscopy was scheduled after $11 \mathrm{am}$ to $12 \mathrm{pm}$. The primary end-point was an equivalence between PMF104 and PEG-ASC in the rate of adequate level of cleansing (Ottawa scale $\leq 6)$, with safety, mucosal visibility, tolerability, acceptance and compliance being also assessed.

Results Of the 403 enrolled, 367 patients (Mean age [SD]: 55.6 (14.4) years; male:166 [45.2\%]) were included in the per protocol (PP) analysis: 184 being randomized in the PMF 104 group and 183 in the PEG-ASC group. Successful bowel cleansing was $78.3 \%$ and $74.3 \%$ in PMF104 and in PEG-ASC, respectively $(P=0.37)$. Both preparations were equally safe (mild adverse events were observed in 9.2\% and $9.3 \%$ of patients in the PMF104 and in the PEG-ASC group, respectively) and acceptable (no or mild distress during the intake in $81.4 \%$ and $80.8 \%$ in the PMF104 in the PEG-ASC, respectively $[P=0.74])$.

Conclusion The new low-volume product Clensia is equivalent to the reference low-volume PEG-ASC in terms of bowel cleansing, safety and acceptance.

EU Clinical Trials Register

2010-019317-22

TRIAL REGISTRATION: Multi-centre, Randomised, Observer-

blind, Comparative Trial EU-CTR 2010-019317-22

at clinicaltrialsregister.eu 


\section{Introduction}

Colonoscopy represents the most accurate investigation for inspecting the mucosa of the colon for diseases such as cancer, adenomas, or inflammation [1]. In particular, it is recommended both for primary colorectal cancer (CRC) screening, and as work-up of organized screening programs with fecal test that are ongoing in several European countries [2,3].

Efficacy of colonoscopy in CRC prevention has been strictly related to its accuracy in detection of precancerous lesions [4, 5].

Quality of inspection, in turn, has been strictly associated with the level of cleansing of the colorectal mucosa [4, 5]. In detail, a substantial miss rate for colorectal neoplasia has been shown when the first of two tandem colonoscopies was performed in patients with inadequate bowel cleansing as compared to those with an adequate level at initial endoscopy [6]. Similarly, implementation of split preparation has been associated with both an improvement in level of cleansing and a higher rate of detection of (advanced) neoplasia and serrate lesions [7].

High-volume polyethylene glycol (PEG)-based solutions have been shown to be highly effective and safe for both outpatient and inpatient bowel preparation, and they are currently recommended as the first-line option by both European and American guidelines [8, 9]. However, the need for $4 \mathrm{~L}$ of PEG affects the overall tolerability and compliance with such solutions [10]. For such reason, an equivalent alternative to these highvolume PEG solutions is represented by low-volume PEG that represents a more balanced compromise between the benefit on one side, and tolerability and patient experience on the other $[8,9]$. These low-volume options frequently exploit a slightly higher osmolarity to compensate for the decrease in total amount of volume. In a recent population-based screening study, the combination of a low-volume PEG and either ascorbate or bisacodyl has been shown to be equivalent to high-volume PEG solutions [11]. In addition, previous meta-analysis showed a substantial equivalence in terms of efficacy between high- and low-volume PEG preparations, while confirming a higher tolerability and compliance with the low-volume solutions $[12,13]$.

PMF104 is a new low-volume bowel preparation in which the synergic osmotic action of PEG, citrates and sulphate compensates for the reduced volume of solution. In addition, simethicone has been included in the formulation to improve the visibility of the colorectal mucosa, as also recognized by the European guidelines. Moreover, to optimize the safety profile, Clensia has been formulated without ascorbate and aspartame, potential harmful components for patients with glucose-6-phosphate dehydrogenase deficiency and phenylketonuria, respectively [8]. In a previous randomized trial, PMF104 has been shown to be equivalent in terms of efficacy and safety as a high-volume PEG, while resulting more tolerable and acceptable to the patients [14]. In detail, successful cleansing was obtained in $73.6 \%$ of patients who received PMF104 as compared to $72.3 \%$ of those who received PEG $4 \mathrm{~L}$. Both regimens were equally safe, but PMF104 showed significantly better gastroin- testinal tolerability compared to PEG 4L: the rate of patients with nausea, bloating, abdominal pain/cramps and anal irritation was lower with PMF104 than with PEG 4L (25.4\% vs. $37.0 \%, P<0.01)$.

The aim of this multicenter, randomized, single-blind study was to compare further the efficacy and safety of PMF104 with those of PEG-ASC that represents a widely used low-volume PEG preparation.

\section{Patients and methods}

\section{Study design}

This was a multicenter, randomized, observer-blind, parallelgroup, phase 3 clinical trial designed to compare a new low-volume PEG-based bowel preparation for colonoscopy (PMF 104) to a standard low-volume PEG-based bowel preparation (PEGASC). The study protocol was reviewed and approved by the Austrian Competent Authority and by the Ethical Committees of the six hospital clinics, respectively located in Graz, Linz, Oberndorf, Ried and Wien (two sites), where the study was carried out. This study was registered in the European Union Clinical Trials Register (EU-CTR: 2010-019317-22). All subjects provided written informed consent.

\section{Study population}

Outpatients of both sexes aged 18 to 85 years and scheduled for routine colonoscopy were considered eligible. Patients with known or suspected hypersensitivity to the product ingredients, known or suspected gastrointestinal obstruction or perforation, toxic megacolon, major colonic resection, end-stage renal insufficiency, phenylketonuria or glucose-6-phosphate dehydrogenase deficiency, or serious diseases that might interfere with the conduct of the study were considered ineligible. Pregnant and breastfeeding women were also excluded.

At enrollment, patients underwent physical examination and samples of blood and urine were collected for clinically relevant laboratory tests. Patients were allocated to receive either the new bowel cleansing agent or the active control according to a computer-generated randomization list (allocation ratio $1: 1)$. These activities were performed by a physician who was not involved in the colonoscopy procedure. This study was observer-blind and the endoscopists performing the colonoscopies were unaware of the treatments assigned to the patients and had to avoid talking with the patients and the staff, who could disclose the type of bowel cleansing agent used.

\section{Bowel cleansing agents}

PMF 104 is a new formulation of PEG-4000 and electrolytes, with citrates and simethicone (Clensia; Alfasigma S.p.A., Milan, Italy) available as powder to be dissolved in $2 \mathrm{~L}$ of water for the preparation of an oral solution.

As active control, a 2-L PEG-3350 solution with ascorbic acid (PEG-ASC) (Moviprep; Norgine Ltd, Harefield, UK) was used.

In accordance with the trial documents, patients in both groups were instructed to take the bowel cleansing agents as full dose (which meant taking the $2 \mathrm{~L}$ of solution plus an additional $1 \mathrm{~L}$ of clear liquids [i.e. water, fruit juice, soft drinks, 
tea]) the evening before the exam for colonoscopies scheduled before $11 \mathrm{am}$ to $12 \mathrm{pm}$, or to take the bowel cleansing agents as split dose, taking the first liter of solution plus half-liter of clear liquids the evening before the exam and the second liter plus another half-liter of clear liquids in the morning of the same day of the procedure for colonoscopies scheduled after $11 \mathrm{am}$ to $12 \mathrm{pm}$. However, after the recommended preparation, ingestion of additional clear liquid was allowed until colonoscopy.

Patients were instructed to follow a low-residue diet during the 3 days before colonoscopy. No solid food was allowed during and after the intake of solution.

\section{Efficacy assessment}

The primary efficacy endpoint was the proportion of patients with successful colon cleansing, defined as "excellent" or "good" according to the Ottawa bowel preparation scale [15]. This is a validated scale (score ranging from 0 to 14) that takes into account two aspects: the degree of segments cleaning and the amount of fluid in the entire colon. Each section of the colon (right, mid and recto-sigmoid colon) is rated according to a 5-point scale $(0-4)$ as follows:

- Excellent: grade $0=$ mucosal detail clearly visible. In case any fluid is present, it is clear. Almost no stool residue;

- Good: grade 1 = some turbid fluid or stool residue but mucosal detail still visible. Washing and suctioning not necessary;

- Fair: grade 2 = turbid fluid or stool residue obscuring mucosal detail. However, mucosal detail becomes visible with suctioning. Washing not necessary;

- Poor: grade 3 = presence of stool obscuring mucosal detail contour. However, with suctioning and washing, a reasonable view is obtained;

- Unprepared: grade 4= solid stool obscuring mucosal detail and contour despite aggressive washing and suctioning.

The overall colonic fluid was rated according to a 3-point scale $(0-2)$ as follows: small=grade 0 ; moderate $=$ grade 1 ; large $=$ grade 2 . The degree of bowel cleansing was categorized according to the total score (sum of single assigned scores) as follows: excellent $(0-3)$, good $(4-6)$, fair $(7-10)$ and inadequate cleansing $(11-14)$. Successful colon cleansing was defined as "excellent" or "good".

Secondary efficacy endpoints included overall mucosal visibility and cecal intubation rate. Mucosal visibility was graded according to a three-point scale (optimal visibility= no or minimal amount of bubbles or foam which can be easily removed; adequate visibility = modest amount of bubble and foam which can be cleared, but requiring loss of time; insufficient visibility = presence of foam and bubbles which significantly reduced the clear visualization of the mucosa) [14]. Cecal intubation rate, defining the completeness of the exam, was assessed as "yes= cecum reached" or "no = cecum not reached".

\section{Safety and tolerability}

The safety of bowel cleansing agents was evaluated by the occurrence of adverse events (AEs) which included abnormal laboratory findings. AEs were monitored throughout the study. Time of onset, duration, severity, outcome, and seriousness of each event were recorded and the causal relation with the study drugs was assessed by the investigators. Standard blood and urine tests were performed at enrollment and at the end of the study.

Occurrence and severity of gastrointestinal symptoms known to be related to intake of bowel cleansing agents such as nausea, bloating, abdominal pain/cramps and anal irritation were included in the gastroinestinal tolerability evaluation.

\section{Acceptability and compliance}

On the day of the procedure, before colonoscopy patients were asked about acceptability (evaluating of the ease of taking the solution and willingness to use the same solution in the further), compliance (evaluated by the amount of drug solution taken) of the bowel cleansing agent and additional clear liquid taken. In particular, patients were asked to assess the ease of taking the solution based on a four-point scale (grade $0=$ no distress; grade 1=mild distress; grade 2=moderate distress; grade 3 =severe distress) and the willingness to use the same bowel cleansing agent in preparation for future exams by answering "yes" or "no".

Compliance was evaluated on a three-point scale: optimal= intake of the whole solution; good = intake of at least $75 \%$ of the solution; poor = intake of less than $75 \%$ of the solution.

The amount of additional clear liquids taken was recorded.

\section{Statistical analysis}

The primary end-point of this trial was demonstration of the equivalence of PMF104 versus PEG-ASC in colon cleansing. An equivalence margin of $15 \%$ was set for the proportion of subjects fulfilling the definition of successful bowel preparation. The null hypothesis was tested by constructing the two-sided $95 \%$ confidence interval $(\mathrm{Cl})$ for the difference in the success rate: the lower limit of the $\mathrm{Cl}$ was compared with the lower equivalence limit of $-15 \%$ and the upper limit of the $\mathrm{Cl}$ was compared with the upper equivalence limit of $15 \%$.

Sample size was calculated a priori based on the estimated rate of $75 \%$ successful cleansing in both treatment groups, a $15 \%$ equivalence margin, a significance level of 0.05 with a power of $84 \%$. Given that $20 \%$ of the enrolled subjects could not be included in the efficacy evaluation (drop-out, major protocol deviations, etc.) at least 204 per group were required.

As recommended by the $\mathrm{ICH}$ guidelines for equivalence study design, efficacy analysis was based on the "per-protocol" (PP) population. Therefore, equivalence testing was limited to the PP population whereas efficacy analysis was performed on both "intention-to-treat" (ITT) and PP population. Statistical analysis of the remaining parameters and the safety analysis were performed on the ITT and safety populations respectively.

PP population included the randomized subjects, who completed the whole study without any major deviations (i. e. viola- 


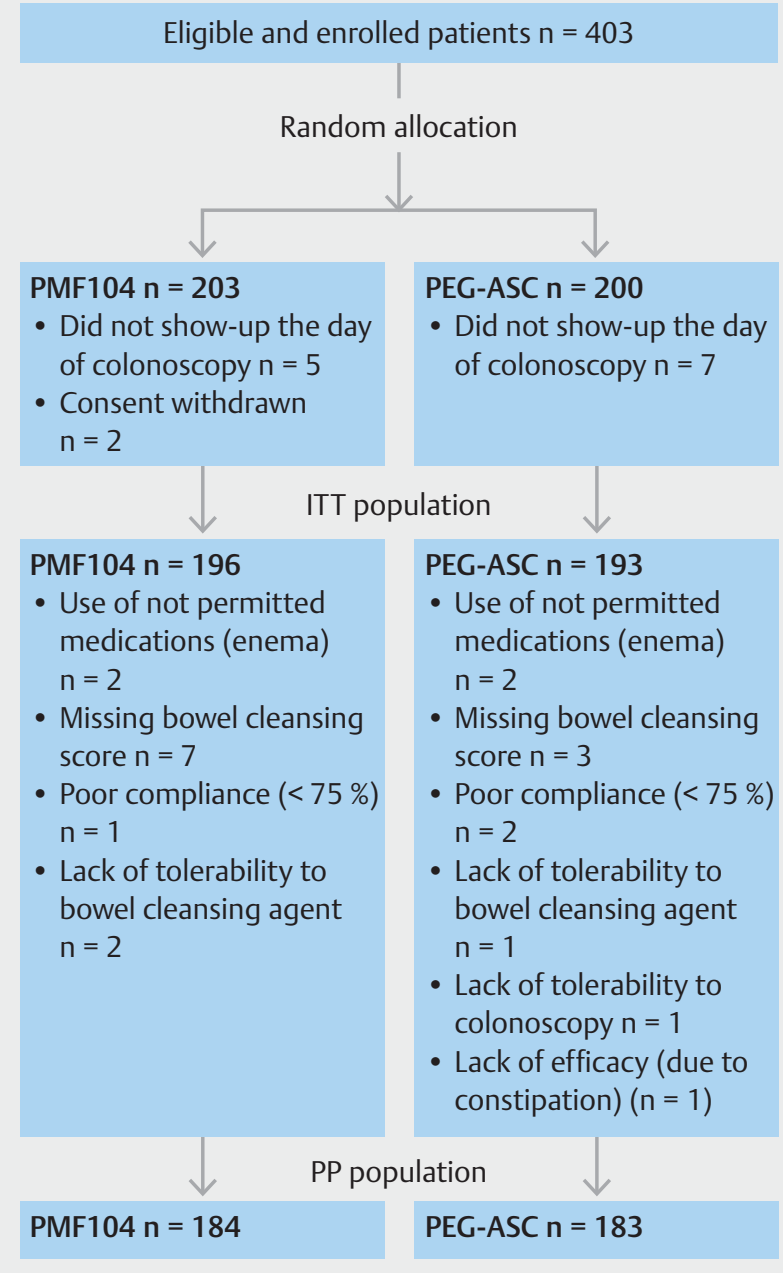

Fig. 1 Patient flow.

tion of inclusion/exclusion criteria, drop-out patients, missing bowel cleansing score data and poor compliance); ITT population included the randomized subjects, who took at least one fraction of the dose of the study formulations and had at least one post-baseline efficacy evaluation; safety population included the randomized subjects, who took at least one fraction of the dose of the study formulations.

Baseline characteristics were summarized using mean and standard deviation (SD) for continuous variables and rates for categorical variables. A two-sided $t$-test was used to compare the means of continuous variables; Chi-squared test was used to compare the rates of categorical measures. The statistical analysis was performed using TESTIMATE Version 6.5 software.

\section{Results}

Patient flow is reported in $>$ Fig.1. Among the 403 enrolled patients, 389 took at least one fraction of the dose of the study formulations and were included in the ITT and safety populations. Twenty-two patients incurred major protocol deviations (10 patients for missed bowel cleansing scores; 4 patients
- Table 1 Demographic characteristics.

\begin{tabular}{|l|c|c|}
\hline & \multicolumn{1}{|l|}{ PMF104 } & PEG-ASC \\
\hline ITT population, $\mathbf{n}$ & 196 & 193 \\
\hline Sex, $\mathrm{n}(\%)$ & & \\
\hline - Male & $93(47.4)$ & $84(43.5)$ \\
\hline - Female & $103(52.6)$ & $109(56.5)$ \\
\hline Age (years), mean (SD) & $54.0(15.1)$ & $56.6(14.1)$ \\
\hline BMI (kg/m²), mean (SD) & $26.2(4.6)$ & $27.2(5.1)$ \\
\hline PP population, n & 184 & 183 \\
\hline Sex, n (\%) & & \\
\hline - Male & $86(46.7)$ & $80(43.7)$ \\
\hline - Female & $98(53.3)$ & $103(56.3)$ \\
\hline Age (years), mean (SD) & $54.3(14.9)$ & $56.8(13.7)$ \\
\hline BMI (kg/m²), mean (SD) & $26.0(4.2)$ & $27.1(5.1)$ \\
\hline SD, standard deviation. & & \\
\hline
\end{tabular}

used not permitted medications such as enemas; 3 patients for poor compliance; 3 patients for lack of tolerance of the bowel cleansing agents; 1 patient for lack of tolerance of colonoscopy; 1 patient for lack of efficacy due to patient severe constipation). Thus, 367 patients (Mean age [SD]: 55.6 [14.35]; male sex [\%]: 166 [45.23]) were included in the PP population. Demographic characteristics of the enrolled patients were similar between groups ( $\triangleright$ Table $\mathbf{1}$ ).

\section{Efficacy}

Overall, the rate of successful bowel cleansing was $78.8 \%$ in PMF 104 and $74.5 \%$ in PEG-ASC for the ITT population ( $P=$ 0.32 ) and $78.3 \%$ in PMF104 and $74.3 \%$ in PEG-ASC for the PP population $(P=0.37)$. In detail, the efficacy of PMF104 was equivalent to PEG-ASC as the $95 \% \mathrm{Cl}$ of the difference between the two groups in the proportion of patients with successful colon cleansing ranged from $-4.8 \%$ to $12.6 \%$ for the PP population and from $-4.2 \%$ to $12.7 \%$ for the ITT population, and it was within the equivalence range -15 to $15 \%$ in both PP and ITT population ( $>$ Table 2 ).

In the ITT population, the rate of successful bowel cleansing was higher with the split-dose regimen (86.2\% for PMF 104 and $78.7 \%$ for PEG-ASC) as compared with the full-dose regimen (75\% for PMF104 and $72.5 \%$ for PEG-ASC) in both groups. The same trend was observed in the PP population.

Similarly, the rate of excellent and good cleansing in the right colon was higher with the split-dose regimen $(73.4 \%$ for PMF104 and $70.5 \%$ for PEG-ASC; $P=0.54$ ) than with the fulldose regimen (52.9\% for PMF104 and 55.1 for PEG-ASC; $P=$ 0.83). The same trend was observed in the PP population.

Mucosa visibility was rated as optimal in $53.9 \%$ of patients in the PMF104 and in $50.5 \%$ of patients in the PEG-ASC group ( $P=$ 0.75). In a subanalysis limited to those who drank up to $1 \mathrm{~L}$ of additional fluid as currently recommended, corresponding to 
- Table2 Bowel cleansing.

\begin{tabular}{|c|c|c|c|}
\hline & $\begin{array}{l}\text { Successful } \\
\text { cleansing } \\
(\%)^{1}\end{array}$ & $\begin{array}{l}\text { Treat- } \\
\text { ment dif- } \\
\text { ference }\end{array}$ & $\begin{array}{l}95 \% \mathrm{Cl} \text { of } \\
\text { treatment } \\
\text { difference }^{2}\end{array}$ \\
\hline \multicolumn{4}{|l|}{ ITT population $n=389$} \\
\hline - PMF104 n= 196 & 78.8 & 4.3 & -4.2 to 12.7 \\
\hline - PEG-ASC $n=193$ & 74.5 & & \\
\hline \multicolumn{4}{|l|}{ PP population $n=367$} \\
\hline - PMF104 n=184 & $78.3 \%$ & 4 & -4.8 to 12.6 \\
\hline - PEG-ASC $n=183$ & $74.3 \%$ & & \\
\hline \multicolumn{4}{|c|}{$\begin{array}{l}\mathrm{Cl} \text {, confidence interval; ITT, intention-to-treat; PP, per-protocol. } \\
1 \text { Successful colon cleansing was considered when the overall OBPS score } \\
\text { was } \leq 6 \text { (excellent and good cleansing) } \\
2 \text { Efficacy was considered equivalent as the } 95 \% \mathrm{CI} \text { for the difference in rates } \\
\text { of successful bowel cleansing between the two treatment groups in the PP } \\
\text { population was entirely included in the range }-15 \text { to } 15 \% \text {. }\end{array}$} \\
\hline
\end{tabular}

$51.3 \%$ of patients in PMF104 and $48.2 \%$ of patients in PEG-ASC groups, respectively, mucosal visibility was rated as optimal in $57.9 \%$ in the PMF104 and in $43.5 \%$ in the PEG-ASC $(P=0.03)$ ( $\triangleright$ Table 3). Optimal mucosa visibility was also higher with the split-dose regimen than with the full-dose regimen in both groups ( $\triangleright$ Table 4 ).

The cecum was reached in $94.8 \%$ of patients in the PMF 104 group and in $96.4 \%$ of patients in the PEG-ASC group $(P=0.46)$.

\section{Safety and tolerability}

No serious adverse event (SAE) occurred during the study and no patient discontinued the study due to AEs. Overall, $9.2 \%$ of patients in the PMF104 and $9.3 \%$ of patients in the PEG-ASC groups experienced an AE. The most frequent AEs were headache $(5.1 \%$ in PMF104 and $1.6 \%$ in PEG-ASC), chills $(0.5 \%$ in PMF104 and $2.1 \%$ in PEG-ASC) and vomiting (0.5\% in PMF104 and $1.6 \%$ in PEG-ASC). No relevant changes to laboratory parameters were observed. The majority of patients reported no distress regarding the gastrointestinal symptoms (nausea, bloating, abdominal pain and anal irritation) with no significant differences between groups with the exception of anal irritation, which was significantly lower in the PMF104 group ( $P=$ 0.03) ( Table 5).

\section{Acceptability and compliance}

The majority of the patients showed no or mild distress during the intake of both bowel cleansing agents $(81.4 \%$ in the PMF104 and $80.8 \%$ in the PEG-ASC; $P=0.74$ ) and reported willingness to take the same solution in case of future colonoscopies (90.2\% in the PMF104 and $90.7 \%$ in the PEG-ASC; $P=0.86$ ).

Optimal compliance was observed in $93.4 \%$ of patients in the PMF104 and in $94.8 \%$ of patients in the PEG-ASC groups ( $P$ $=0.75)$.

\section{Discussion}

According to the current randomized study, the new low-volume PEG solution with citrate and simethicone is equivalent in terms of cleansing quality and safety to the reference low-volume preparation, represented by the combination of PEG and ascorbate. In addition, both solutions appear to be well-tolerated and accepted by patients.

The results of the study are notable for the following reasons. It could be shown that two low-volume solutions with a slight hyperosmolarity by contribution of two different additional molecules, citrate and ascorbate, were equally effective in reaching an adequate level of cleansing. Thus, the effect related to hyperosmolarity appeared to be independent of the molecular agent. Of note, Clensia hyperosmolality is slightly lower than that of PEG-ASC used in the current trial $(450 \mathrm{mOs}$ $\mathrm{mol} / \mathrm{kg}$ vs. $553 \mathrm{mOsmol} / \mathrm{kg}$ ), potentially favoring its safety profile. Second, both of the regimens were substantially more effective used by split-dose intake as compared with a non-split regimen. In particular, a very high rate of adequate cleansing was achieved with both regimens in a split regimen, namely $87 \%$ and $78 \%$. It could be argued that the $87 \%$ reached by Clensia is still lower than the $90 \%$ required by European and American guidelines [4,5]. However, it should be noted that Ottawa scale was used, which does not allow for intra-procedural cleansing (washing and suctioning maneuvers). Therefore, the effects of a study preparation itself was better evaluated because the degree of bowel cleansing was that achieved by the bowel preparation regimen [15], In addition, the current data confirm the advantage of the split-dose regimen for cleansing quality of the proximal colon. This is a critical point as adequate cleansing has been shown to facilitate identification of serrated adenomas which tend to be flat and difficult to detect [16].

- Table 3 Mucosa visibility in relation to the amount of additional liquids taken by the patients.

\begin{tabular}{|l|l|l|l|}
\hline & ITT population & & P value \\
\hline Overall & PMF $104 \mathrm{n}=191$ & PEG-ASC $n=192$ \\
\hline - Optimal visibility, $\mathrm{n}(\%)$ & $103(53.9)$ & $97(50.5)$ & 0.75 \\
\hline Up to 1 liter & PMF $104 \mathrm{n}=95$ & PEG-ASC $n=92$ & 0.03 \\
\hline - Optimal visibility, $n(\%)$ & $55(57.9)$ & $40(43.5)$ & \\
\hline ITT, intention-to-treat. & & \\
\hline
\end{tabular}


- Table 4 Mucosa visibility.

\begin{tabular}{|l|l|l|l|}
\hline & \multicolumn{2}{|c|}{ Optimal visibility } & \\
\hline & $\begin{array}{l}\text { Split dose } \\
\text { regimen (\%) }\end{array}$ & $\begin{array}{l}\text { Full dose re- } \\
\text { gimen (\%) }\end{array}$ & P value \\
\hline ITT population & & & \\
\hline - PMF $104 \mathrm{n}=191$ & 60 & 50.8 & 0.07 \\
\hline - PEG-ASC $\mathrm{n}=192$ & 65.6 & 43.5 & 0.01 \\
\hline ITT, intention-to-treat. & & & \\
\hline
\end{tabular}

Third, no issues related to safety emerged in the current study. In particular, electrolyte measurement before and after preparation excluded clinically relevant alterations in sodium and potassium homeostasis, offering reassurance as to its safety as a laxative for outpatient colonoscopy. Fourth, the rate of optimal mucosal visibility was similar between groups. To note, in the current trial, there was no fluid intake restriction during preparation and additional ingestion of clear liquids was allowed, also exceeding the volumes recommended in the protocol instructions for the two products (i. e. $1 \mathrm{~L}$ ). Interestingly, when considering the group of participants limiting the additional fluid to $1 \mathrm{~L}$ (i.e. in accordance with the amount recommended by the protocol instructions), mucosal visibility was higher $(P<0.03)$ in patients receiving Clensia. Although a definite explanation is not clear, this result may be related to the anti-foaming effect of simethicone that in presence of lower amounts of water might result in greater efficacy. Fifth, the high tolerability and acceptance of low-volume preparations for colonoscopy further support the need to implement such regimens in screening programs to improve the overall perception of such programs within the target population.

The clinical relevance of the current study must be integrated with the main results of a previous comparison between Clensia and a high-volume 4-L PEG preparation [14]. In detail, an equivalence in terms of efficacy and safety between these two regimens was shown, while the low-volume Clensia resulting - as expected - was better tolerated and accepted. Thus, Clensia appeared equivalent to both the current available options of low- and high-volume PEG-based bowel cleansing agents. It may be included among the possible options for outpatient colonoscopy with emphasis on the absence of potentially harmful components in its formulation.

This study has some limitations. Polyp and adenoma detection rates (ADR), which are one of the main outcomes of colonoscopies, were not collected. Therefore, with reference to these parameters, it is not possible to state whether there is a difference between the study formulations. On the other hand, all existing related data are showing a positive correlation between cleansing quality and ADR. Thus, there is no reason for an adverse supposition for this trial. However, rates of detection of polyps and adenomas may be affected by other variables such as an endoscopist's technique and technology that may confound the results. Second, the association between risk factors for inadequate bowel preparation such as male sex, older
- Table 5 Gastrointestinal tolerability.

\begin{tabular}{|l|l|l|l|}
\hline PMF104 & PEG-ASC & P value \\
& $n=194$ & $n=193$ & \\
\hline
\end{tabular}

No gastrointestinal distress, $\mathrm{n}(\%)$

\begin{tabular}{|l|l|l|l|}
\hline - Nausea & $168(86.6)$ & $167(86.5)$ & 0.85 \\
\hline - Bloating & $168(86.6)$ & $165(85.5)$ & 0.80 \\
\hline - Abdominal pain & $160(82.5)$ & $156(80.8)$ & 0.59 \\
\hline - Anal irritation & $184(94.9)$ & $168(87.1)$ & 0.03 \\
\hline
\end{tabular}

age, overweight or presence of comorbidities (e.g. cirrhosis, diabetes, or Parkinson's disease requiring therapy) and level of bowel cleansing and mucosa visibility has not been investigated. Therefore, the effects of the two bowel cleansing agents on patients at risk for inadequate preparation remain unknown. Furthermore, previous patient experience with a high-volume bowel preparation might favor the patient's acceptance of a low-volume preparation. This aspect has not been assessed. Finally, another limitation is that the mucosa visibility scale used in this study has not been validated and the scores attributed by endoscopists may be subject to interobserver variability.

\section{Conclusion}

In conclusion, the low-volume preparation Clensia is equivalent to PEG-ASC in terms of bowel cleansing, safety and acceptability. The rate of successful bowel cleansing was higher with the split-dose regimen compared to the full-dose regimen in both groups. Specifically designed studies are required to better define the exact role of simethicone in bowel cleansing.

\section{Competing interests}

Cesare Hassan and Cristiano Spada received consultation fees from Alfasigma.

\section{References}

[1] Lee T], Nair S, Beintaris I et al. Recent advances in colonoscopy. F1000Res 2016; 11;5: 328

[2] Rex DK, Johnson DA, Anderson JC et al. American College of Gastroenterology guidelines for colorectal cancer screening 2009 [corrected]. Am J Gastroenterol 2009; 104: 739-750

[3] von Karsa L, Patnick J, Segnan $\mathrm{N}$ et al. European guidelines for quality assurance in colorectal cancer screening and diagnosis: overview and introduction to the full supplement publication. Endoscopy 2013; 45: $51-59$

[4] Rex DK, Schoenfeld PS, Cohen J et al. Quality indicators for colonoscopy. Gastrointest Endosc 2015; 81: 31 - 53

[5] Kaminski MF, Thomas-Gibson S, Bugajski M et al. Performance measures for lower gastrointestinal endoscopy: a European Society of Gastrointestinal Endoscopy (ESGE) Quality Improvement Initiative. Endoscopy 2017; 49: $378-397$ 
[6] Clark BT, Protiva P, Nagar A et al. Quantification of Adequate Bowel Preparation for Screening or Surveillance Colonoscopy in Men. Gastroenterology 2016; 150: 396 - 405; quiz e14-15

[7] Radaelli F, Paggi S, Hassan C et al. Split-dose preparation for colonoscopy increases adenoma detection rate: a randomised controlled trial in an organised screening programme. Gut 2017; 66: $270-277$

[8] Hassan C, Bretthauer M, Kaminski MF et al. Bowel preparation for coIonoscopy: European Society of Gastrointestinal Endoscopy (ESGE) guideline. Endoscopy 2013; 45: 142 - 150

[9] Johnson DA, Barkun AN, Cohen LB et al. Optimizing adequacy of bowel cleansing for colonoscopy: recommendations from the US multi-society task force on colorectal cancer. Gastroenterology 2014; 147: $903-924$

[10] Bucci C, Rotondano G, Hassan C et al. Optimal bowel cleansing for colonoscopy: split the dose! A series of meta-analyses of controlled studies. Gastrointest Endosc 2014; 80: 566- 576

[11] Zorzi M, Valiante F, Germanà B et al. Comparison between different colon cleansing products for screening colonoscopy. A noninferiority trial in population-based screening programs in Italy. Endoscopy 2016; 48: $223-231$

[12] Martel M, Barkun AN, Menard C et al. Split-Dose Preparations Are Superior to Day-Before Bowel Cleansing Regimens: A Meta-analysis. Gastroenterology 2015; 149: 79-88

[13] Xie Q, Chen L, Zhao F et al. A meta-analysis of randomized controlled trials of low-volume polyethylene glycol plus ascorbic acid versus standard-volume polyethylene glycol solution as bowel preparations for colonoscopy. PloS One 2014; 9: e99092

[14] Spada C, Cesaro P, Bazzoli F et al. Evaluation of Clensia ${ }^{\circledR}$, a new lowvolume PEG bowel preparation in colonoscopy: Multicentre randomized controlled trial versus 4L PEG. Dig Liver Dis 2017; 49: 651-656

[15] Rostom A, Jolicoeur E. Validation of a new scale for the assessment of bowel preparation quality. Gastrointest Endosc 2004; 59: 482 - 486

[16] Rex DK, Ahnen DJ, Baron JA et al. Serrated lesions of the colorectum: review and recommendations from an expert panel. Am J Gastroenterol 2012; 107: 1315 -1329; quiz 1314-1330 